Supporting information

\title{
Sensing Mechanism of a Fluorescent Probe for Cysteine: Photo-Induced Electron Transfer and Invalidity of Excited-State Intramolecular Proton Transfer
}

Zhe Tang ${ }^{\mathrm{ab}}$, Tianxin Baiab, Panwang Zhou ${ }^{\mathrm{a}^{*}}$

${ }^{a}$ Institute of Molecular Sciences and Engineering, Institute of Frontier and Interdisciplinary Science, Shandong University, Qingdao 266237, P.R. China

b State Key Laboratory of Molecular Reaction Dynamics, Dalian Institute of Chemical Physics, Chinese Academy of Sciences, Dalian 116023, P. R. China

E-mail: pwzhou@,sdu.edu.cn

Tel: $+86-532-58632158$ 
Table S1. Comparison of experimental and calculated emission for MZC in the PBE0 and B3LYP functionals.

\begin{tabular}{|c|c|c|c|c|c|c|c|c|}
\hline & & & $\begin{array}{l}\text { electronic } \\
\text { transition }\end{array}$ & $\begin{array}{c}\text { energy }(\mathrm{nm} \\
/ \mathrm{eV})\end{array}$ & $f$ & contrib & $\mathrm{CI}$ & $\begin{array}{c}\exp \\
(\mathrm{nm} / \mathrm{eV})\end{array}$ \\
\hline \multirow{2}{*}{ PBE0 } & MZC-enol & \multirow{2}{*}{ emission } & $\mathrm{S}_{1} \rightarrow \mathrm{S}_{0}$ & $472 / 2.63$ & 0.3457 & $\mathrm{H} \rightarrow \mathrm{L}$ & 0.99 & \multirow{4}{*}{$475 / 2.6105$} \\
\hline & MZC-keto & & $\mathrm{S}_{1} \rightarrow \mathrm{S}_{0}$ & $538 / 2.32$ & 0.3932 & $\mathrm{H} \rightarrow \mathrm{L}$ & 0.99 & \\
\hline \multirow{2}{*}{ B3LYP } & MZC-enol & \multirow{2}{*}{ emission } & $\mathrm{S}_{1} \rightarrow \mathrm{S}_{0}$ & $487 / 2.55$ & 0.3219 & $\mathrm{H} \rightarrow \mathrm{L}$ & 0.99 & \\
\hline & MZC-keto & & $\mathrm{S}_{1} \rightarrow \mathrm{S}_{0}$ & $558 / 2.22$ & 0.3470 & $\mathrm{H} \rightarrow \mathrm{L}$ & 0.99 & \\
\hline
\end{tabular}

Figure S1. Computed energy profiles along the constructed LIIC pathways between the optimized $\mathrm{S}_{0}$ and CT (right) and LE (left) states. 

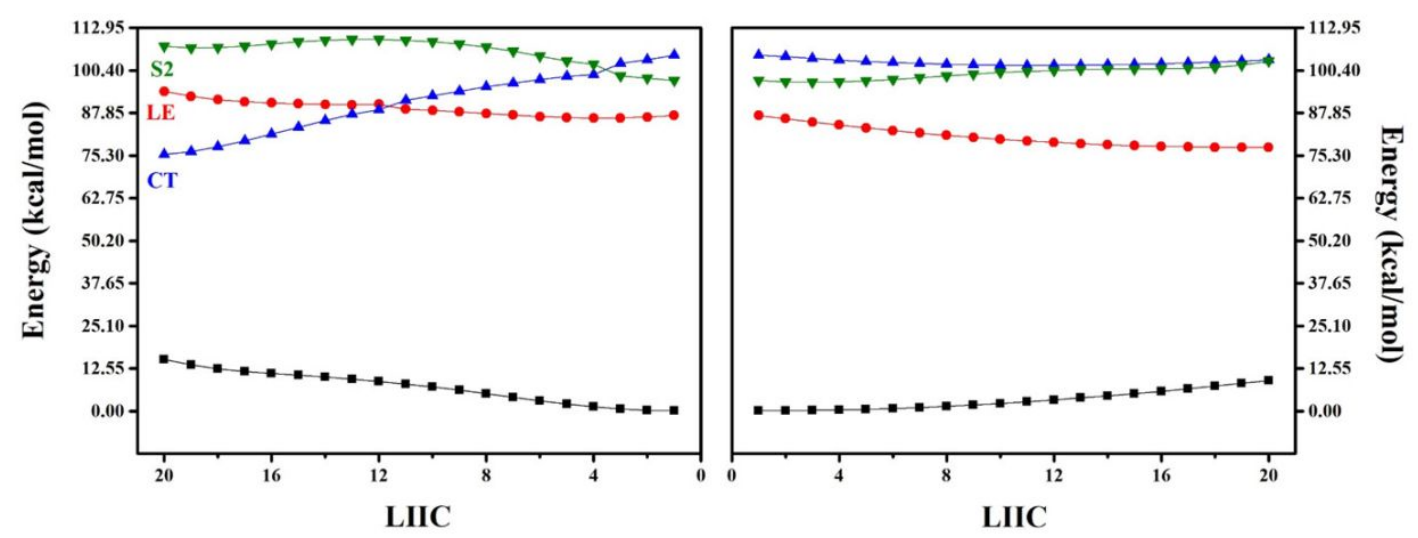

Figure S2. Calculated IR spectra of MZC-enol at the spectral region of $\mathrm{O}_{1}-\mathrm{H}_{2}$ stretching band in the $\mathrm{S}_{0}$ and $\mathrm{S}_{1}$ states based on CAM-B3LYP/TZVP/IEFPCM levels.

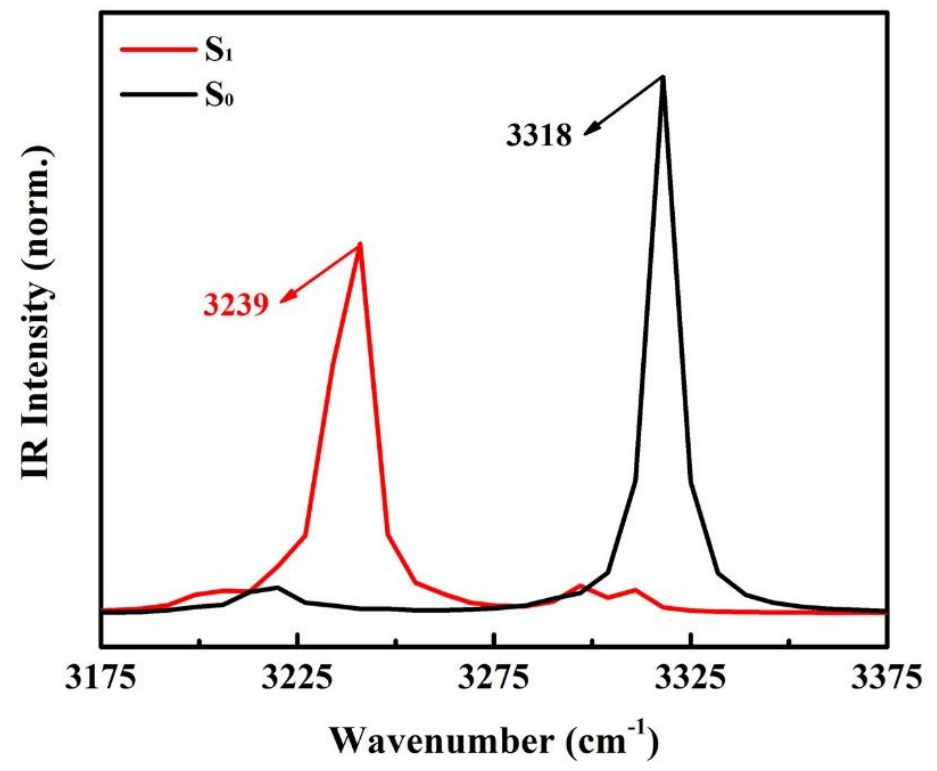

$\mathrm{S}_{0}$ geometry of MZC-AC in water solvent, cam-b3lyp/tzvp/iefpcm, empiricaldispersion $=\mathrm{gd} 3 \mathrm{bj}$

Energy $=-1107.740012$

$\begin{array}{lrrr}\mathrm{C} & 1.00082100 & -1.92676200 & -0.09899100 \\ \mathrm{C} & 1.70513400 & -3.13184800 & -0.34220400 \\ \mathrm{C} & 1.02053600 & -4.26493700 & -0.63021200 \\ \mathrm{C} & -0.40503000 & -4.24399700 & -0.70169500 \\ \mathrm{C} & -1.08814000 & -3.11402900 & -0.43925300 \\ \mathrm{C} & -0.83771000 & -0.70736500 & 0.21022300 \\ \mathrm{C} & 1.32702900 & -0.61248800 & 0.20282300 \\ \mathrm{H} & 2.78326000 & -3.12832600 & -0.28544800\end{array}$




\begin{tabular}{|c|c|c|c|}
\hline $\mathrm{H}$ & 1.54767100 & -5.19089000 & -0.81260900 \\
\hline $\mathrm{H}$ & -0.95274400 & -5.13724400 & -0.96405800 \\
\hline $\mathrm{H}$ & -2.16233500 & -3.03497800 & -0.47928200 \\
\hline $\mathrm{C}$ & -2.24962900 & -0.32893200 & 0.31840900 \\
\hline $\mathrm{C}$ & -3.15797200 & -1.08984400 & 1.05319900 \\
\hline $\mathrm{C}$ & -2.72340600 & 0.82756100 & -0.29620800 \\
\hline $\mathrm{C}$ & -4.48542200 & -0.71254000 & 1.15660900 \\
\hline $\mathrm{H}$ & -2.81298800 & -1.97764400 & 1.56648200 \\
\hline $\mathrm{C}$ & -4.04401300 & 1.21485200 & -0.20137600 \\
\hline $\mathrm{C}$ & -4.93104900 & 0.43972000 & 0.52713100 \\
\hline $\mathrm{H}$ & -5.17127500 & -1.31683800 & 1.73493300 \\
\hline $\mathrm{H}$ & -4.36575200 & 2.11674400 & -0.70412200 \\
\hline $\mathrm{H}$ & -5.96775400 & 0.73872400 & 0.60434400 \\
\hline $\mathrm{C}$ & 2.64494700 & 0.01224400 & 0.33825800 \\
\hline $\mathrm{C}$ & 3.74831400 & -0.43177300 & -0.39103500 \\
\hline $\mathrm{C}$ & 2.81077200 & 1.09958600 & 1.19817500 \\
\hline $\mathrm{C}$ & 4.98401300 & 0.17953500 & -0.24824800 \\
\hline $\mathrm{H}$ & 3.63803800 & -1.24158500 & -1.09926800 \\
\hline $\mathrm{C}$ & 4.04340000 & 1.71427700 & 1.33287200 \\
\hline $\mathrm{H}$ & 1.95879000 & 1.45774300 & 1.76022700 \\
\hline $\mathrm{C}$ & 5.13856000 & 1.25357500 & 0.61484000 \\
\hline $\mathrm{H}$ & 5.82715600 & -0.17839500 & -0.82539100 \\
\hline $\mathrm{H}$ & 4.15188900 & 2.55533200 & 2.00605800 \\
\hline $\mathrm{H}$ & 6.10313100 & 1.73252400 & 0.72280800 \\
\hline $\mathrm{N}$ & 0.18353300 & 0.09915300 & 0.39129200 \\
\hline $\mathrm{O}$ & -1.85605900 & 1.57067600 & -1.08883900 \\
\hline $\mathrm{N}$ & -0.39713600 & -1.96736400 & -0.10395900 \\
\hline $\mathrm{C}$ & -1.21367300 & 2.62256000 & -0.52243800 \\
\hline $\mathrm{O}$ & -1.46295500 & 3.03154300 & 0.57976600 \\
\hline $\mathrm{C}$ & -0.21038000 & 3.17148400 & -1.45442200 \\
\hline $\mathrm{H}$ & -0.13195400 & 2.69309400 & -2.42125400 \\
\hline $\mathrm{C}$ & 0.56100100 & 4.18639400 & -1.10076000 \\
\hline $\mathrm{H}$ & 0.46649400 & 4.64138400 & -0.12266300 \\
\hline $\mathrm{H}$ & 1.30710000 & 4.58644500 & -1.77368100 \\
\hline
\end{tabular}

LE geometry of MZC-AC in water solvent, cam-b3lyp/tzvp/iefpcm, empiricaldispersion $=\mathrm{gd} 3 \mathrm{bj}$

Energy $=-1107.620472$

$\begin{array}{cccc}\mathrm{C} & 0.98520800 & 2.01795400 & 0.09942800 \\ \mathrm{C} & 1.65504300 & 3.20309500 & 0.30335700 \\ \mathrm{C} & 0.89192400 & 4.38048500 & 0.60230300 \\ \mathrm{C} & -0.47444400 & 4.30390100 & 0.71745300\end{array}$




\begin{tabular}{lrrc}
$\mathrm{C}$ & -1.15038600 & 3.10551600 & 0.50991700 \\
$\mathrm{C}$ & -0.82115800 & 0.70552900 & -0.14114400 \\
$\mathrm{C}$ & 1.33682700 & 0.64198600 & -0.18395800 \\
$\mathrm{H}$ & 2.72555300 & 3.26073100 & 0.23431300 \\
$\mathrm{H}$ & 1.40446500 & 5.31887000 & 0.75887300 \\
$\mathrm{H}$ & -1.05708600 & 5.17561800 & 0.98200900 \\
$\mathrm{H}$ & -2.20700000 & 2.96146700 & 0.64683600 \\
$\mathrm{C}$ & -2.22249500 & 0.32264600 & -0.26918700 \\
$\mathrm{C}$ & -3.12912800 & 1.11275000 & -0.97829000 \\
$\mathrm{C}$ & -2.68319200 & -0.87734200 & 0.27321400 \\
$\mathrm{C}$ & -4.44561600 & 0.71773800 & -1.12816200 \\
$\mathrm{H}$ & -2.78822300 & 2.03191900 & -1.43435700 \\
$\mathrm{C}$ & -3.99663400 & -1.27243000 & 0.13849100 \\
$\mathrm{C}$ & -4.88180400 & -0.47211100 & -0.56577400 \\
$\mathrm{H}$ & -5.13122300 & 1.33908200 & -1.68792500 \\
$\mathrm{H}$ & -4.31512800 & -2.20193400 & 0.58998500 \\
$\mathrm{H}$ & -5.91224300 & -0.78192300 & -0.67631900 \\
$\mathrm{C}$ & 2.61669300 & 0.02555400 & -0.30417300 \\
$\mathrm{C}$ & 3.82897300 & 0.70859000 & -0.07565900 \\
$\mathrm{C}$ & 2.68717300 & -1.34263500 & -0.65560800 \\
$\mathrm{C}$ & 5.04000300 & 0.05623500 & -0.20132000 \\
$\mathrm{H}$ & 3.82789700 & 1.74273900 & 0.22372500 \\
$\mathrm{C}$ & 3.90029000 & -1.97865800 & -0.78121100 \\
$\mathrm{H}$ & 1.76697600 & -1.88280200 & -0.82239200 \\
$\mathrm{C}$ & 5.08851800 & -1.28481400 & -0.55795800 \\
$\mathrm{H}$ & 5.95761100 & 0.59966100 & -0.01609000 \\
$\mathrm{H}$ & 3.92937300 & -3.02598300 & -1.05357000 \\
$\mathrm{H}$ & 6.04118200 & -1.78826700 & -0.65774600 \\
$\mathrm{~N}$ & 0.19020600 & -0.09975100 & -0.32245900 \\
$\mathrm{O}$ & -1.82205700 & -1.64811700 & 1.04085200 \\
$\mathrm{~N}$ & -0.39620200 & 1.99922300 & 0.14962500 \\
$\mathrm{C}$ & -1.15367200 & -2.66260600 & 0.43183000 \\
$\mathrm{O}$ & -1.36558100 & -3.00203300 & -0.70053700 \\
$\mathrm{C}$ & -0.17993900 & -3.26294700 & 1.36202400 \\
$\mathrm{H}$ & -0.13280700 & -2.84056900 & 2.35649800 \\
$\mathrm{C}$ & 0.60231900 & -4.25724100 & 0.97540900 \\
$\mathrm{H}$ & 0.53922800 & -4.65534400 & -0.02968700 \\
$\mathrm{H}$ & 1.32749000 & -4.69553500 & 1.64727600 \\
& & & \\
\hline
\end{tabular}

CT geometry of MZC-AC in water solvent, cam-b3lyp/tzvp/iefpcm, empiricaldispersion $=\mathrm{gd} 3 \mathrm{bj}$

Energy $=-1107.619828$ 


\begin{tabular}{|c|c|c|c|}
\hline $\mathrm{C}$ & 0.90311900 & 1.01258200 & -0.79171000 \\
\hline $\mathrm{C}$ & 1.44216300 & 2.28354600 & -0.86338600 \\
\hline $\mathrm{C}$ & 0.59894700 & 3.34407200 & -1.15250800 \\
\hline $\mathrm{C}$ & -0.76260600 & 3.13319600 & -1.37497400 \\
\hline $\mathrm{C}$ & -1.28691000 & 1.87509000 & -1.26150900 \\
\hline $\mathrm{C}$ & -0.75283500 & -0.48852000 & -0.68579700 \\
\hline $\mathrm{C}$ & 1.38910400 & -0.31445600 & -0.54077400 \\
\hline $\mathrm{H}$ & 2.49453500 & 2.44385300 & -0.69858600 \\
\hline $\mathrm{H}$ & 1.00351500 & 4.34379600 & -1.21678700 \\
\hline $\mathrm{H}$ & -1.41629600 & 3.95337300 & -1.62944600 \\
\hline $\mathrm{H}$ & -2.32714400 & 1.62992500 & -1.39940100 \\
\hline $\mathrm{C}$ & -2.11400400 & -0.96592800 & -0.54487600 \\
\hline $\mathrm{C}$ & -2.55629300 & -2.07346400 & -1.26637900 \\
\hline $\mathrm{C}$ & -2.98107800 & -0.32803900 & 0.35740500 \\
\hline $\mathrm{C}$ & -3.86215200 & -2.50438400 & -1.15149400 \\
\hline $\mathrm{H}$ & -1.86713500 & -2.57138400 & -1.93570700 \\
\hline $\mathrm{C}$ & -4.29389700 & -0.76396500 & 0.45992700 \\
\hline $\mathrm{C}$ & -4.73257700 & -1.83454800 & -0.29753300 \\
\hline $\mathrm{H}$ & -4.20614600 & -3.35236800 & -1.72745200 \\
\hline $\mathrm{H}$ & -4.95113400 & -0.26226200 & 1.15719700 \\
\hline $\mathrm{H}$ & -5.75936500 & -2.16434900 & -0.20726500 \\
\hline $\mathrm{C}$ & 2.74354800 & -0.74798600 & -0.29084600 \\
\hline $\mathrm{C}$ & 3.85221700 & -0.02637100 & -0.74644500 \\
\hline $\mathrm{C}$ & 2.95109400 & -1.92600300 & 0.43798500 \\
\hline $\mathrm{C}$ & 5.13184100 & -0.46915600 & -0.47210300 \\
\hline $\mathrm{H}$ & 3.71903700 & 0.85613300 & -1.35441200 \\
\hline $\mathrm{C}$ & 4.23261300 & -2.35755300 & 0.71521200 \\
\hline $\mathrm{H}$ & 2.09474700 & -2.48182400 & 0.79243500 \\
\hline $\mathrm{C}$ & 5.32609300 & -1.63001400 & 0.26375600 \\
\hline $\mathrm{H}$ & 5.98234100 & 0.08898600 & -0.83979200 \\
\hline $\mathrm{H}$ & 4.38283700 & -3.26280700 & 1.28816900 \\
\hline $\mathrm{H}$ & 6.32993400 & -1.97068300 & 0.48061800 \\
\hline $\mathrm{N}$ & 0.34971700 & -1.17274800 & -0.51196100 \\
\hline $\mathrm{O}$ & -2.57664900 & 0.71366200 & 1.12890700 \\
\hline $\mathrm{N}$ & -0.45441300 & 0.86042900 & -0.93323800 \\
\hline $\mathrm{C}$ & -1.32610000 & 0.56799700 & 1.85501000 \\
\hline $\mathrm{O}$ & -0.99099700 & -0.57068600 & 2.21073900 \\
\hline $\mathrm{C}$ & -0.67186000 & 1.79626800 & 2.01605300 \\
\hline $\mathrm{H}$ & -1.18511800 & 2.66459500 & 1.62413800 \\
\hline $\mathrm{C}$ & 0.57732500 & 1.93855100 & 2.56979200 \\
\hline $\mathrm{H}$ & 1.10757600 & 1.08818300 & 2.97426500 \\
\hline $\mathrm{H}$ & 1.02533900 & 2.91377300 & 2.68241300 \\
\hline
\end{tabular}


$\mathrm{S}_{0}$ geometry of MZC-enol in water solvent, cam-b3lyp/tzvp/iefpcm, empiricaldispersion $=\mathrm{gd} 3 \mathrm{bj}$

Energy $=-917.029932$

$\begin{array}{lrrr}\mathrm{C} & 0.83322400 & 1.40165100 & 0.02898600 \\ \mathrm{C} & 1.51782800 & 2.64160900 & 0.04133300 \\ \mathrm{C} & 0.81716300 & 3.79722800 & -0.03578200 \\ \mathrm{C} & -0.60504300 & 3.76221300 & -0.15203600 \\ \mathrm{C} & -1.27143700 & 2.59369600 & -0.11997000 \\ \mathrm{C} & -0.98313000 & 0.10704600 & 0.08927300 \\ \mathrm{C} & 1.19890000 & 0.06712700 & 0.05582100 \\ \mathrm{H} & 2.59490200 & 2.64045200 & 0.11700900 \\ \mathrm{H} & 1.32700200 & 4.75020100 & -0.02303400 \\ \mathrm{H} & -1.16636200 & 4.67750000 & -0.26998300 \\ \mathrm{H} & -2.33926700 & 2.51571600 & -0.22483500 \\ \mathrm{C} & -2.35423100 & -0.39575600 & 0.09761800 \\ \mathrm{C} & -3.43065100 & 0.32031600 & 0.62549300 \\ \mathrm{C} & -2.60112200 & -1.68535300 & -0.41559300 \\ \mathrm{C} & -4.71927200 & -0.17745800 & 0.58468100 \\ \mathrm{H} & -3.25630300 & 1.27259400 & 1.10481300 \\ \mathrm{C} & -3.90070200 & -2.17296600 & -0.47024200 \\ \mathrm{C} & -4.95355500 & -1.42329600 & 0.01861200 \\ \mathrm{H} & -5.53377500 & 0.39920500 & 1.00122600 \\ \mathrm{H} & -4.05998400 & -3.15915300 & -0.88604900 \\ \mathrm{H} & -5.95903500 & -1.82140300 & -0.02528900 \\ \mathrm{C} & 2.53427800 & -0.53297400 & 0.07139500 \\ \mathrm{C} & -1.61012500 & -2.48658000 & -0.86413000 \\ \mathrm{C} & -0.74782200 & -2.08506700 & -0.59864000 \\ \mathrm{C} & -0.56863000 & 1.41089300 & 0.02359300 \\ \mathrm{H} & 2.61231500 & 0.06437500 & -0.58194900 \\ \mathrm{C} & 2.74435600 & -1.74080600 & 0.73845900 \\ \mathrm{H} & 4.86844100 & -0.51997300 & -0.55154500 \\ \mathrm{C} & 3.46533200 & 0.97681400 & -1.14371400 \\ \mathrm{H} & 3.99794300 & -2.32718700 & 0.76007500 \\ \mathrm{H} & 1.91363600 & -2.21429300 & 1.24460500 \\ \mathrm{H} & 5.06768400 & -1.71648300 & 0.12026300 \\ \mathrm{~N} & 5.69248200 & -0.04314300 & -1.06680400 \\ \mathrm{O} & 4.14282200 & -3.26316600 & 1.28453200 \\ \mathrm{H} & 6.04851800 & -2.17357800 & 0.14048800 \\ \mathrm{~N} & 0.07161800 & -0.69062000 & 0.09488800 \\ & & & \\ & & & \end{array}$

$\mathrm{S}_{1}$ geometry of MZC-enol in water solvent, cam-b3lyp/tzvp/iefpcm, 
empiricaldispersion $=\mathrm{gd} 3 \mathrm{bj}$

Energy $=-916.912146$

$\begin{array}{lrrr}\mathrm{C} & 0.82640900 & 1.46715800 & 0.02409300 \\ \mathrm{C} & 1.47986900 & 2.67894000 & 0.05922500 \\ \mathrm{C} & 0.70828300 & 3.87923700 & -0.04187500 \\ \mathrm{C} & -0.65000100 & 3.80543800 & -0.22141800 \\ \mathrm{C} & -1.31119400 & 2.57986100 & -0.24776200 \\ \mathrm{C} & -0.96301200 & 0.10743300 & 0.04793300 \\ \mathrm{C} & 1.21529500 & 0.07863000 & 0.06259200 \\ \mathrm{H} & 2.54688700 & 2.72887700 & 0.17834700 \\ \mathrm{H} & 1.20787900 & 4.83691900 & -0.00907000 \\ \mathrm{H} & -1.24071300 & 4.70048400 & -0.35936600 \\ \mathrm{H} & -2.34901300 & 2.45960700 & -0.49462900 \\ \mathrm{C} & -2.31519600 & -0.39656800 & 0.08451100 \\ \mathrm{C} & -3.40827700 & 0.35832400 & 0.53735700 \\ \mathrm{C} & -2.54437100 & -1.73856200 & -0.30799500 \\ \mathrm{C} & -4.68423200 & -0.15578500 & 0.53627100 \\ \mathrm{H} & -3.24107300 & 1.35026400 & 0.92905600 \\ \mathrm{C} & -3.84343400 & -2.23613700 & -0.33202400 \\ \mathrm{C} & -4.90079200 & -1.45423500 & 0.08066300 \\ \mathrm{H} & -5.50873600 & 0.44230600 & 0.89845500 \\ \mathrm{H} & -3.99261000 & -3.25627600 & -0.65912000 \\ \mathrm{H} & -5.90274000 & -1.86308700 & 0.06730500 \\ \mathrm{C} & 2.51368800 & -0.50967100 & 0.07160100 \\ \mathrm{C} & 3.69083700 & 0.22567200 & -0.17667800 \\ \mathrm{C} & 2.64222900 & -1.89506000 & 0.32219400 \\ \mathrm{C} & -1.55483500 & -0.39408800 & -0.16376500 \\ \mathrm{H} & -0.69073500 & -2.12351000 & -0.47552000 \\ \mathrm{C} & 3.63858500 & 1.27412700 & -0.41995200 \\ \mathrm{H} & 3.87918700 & -2.49792900 & 0.34091100 \\ \mathrm{C} & 1.75259500 & -2.47716300 & 0.51514300 \\ \mathrm{H} & 5.03223300 & -1.75326000 & 0.10102800 \\ \mathrm{H} & 5.81385300 & 0.18864900 & -0.36767100 \\ \mathrm{H} & 3.95410400 & -3.55833000 & 0.54518100 \\ \mathrm{~N} & 6.00303100 & -2.23095400 & 0.11654500 \\ \mathrm{O} & 0.08699500 & -0.69464700 & 0.08420800 \\ \mathrm{~N} & -2.56717000 & -0.67470000 \\ & -1.43308600 & -0.04538900\end{array}$

$\mathrm{S}_{1}$ geometry of MZC-keto in water solvent, cam-b3lyp/tzvp/iefpcm, empiricaldispersion $=\mathrm{gd} 3 \mathrm{bj}$ 


\begin{tabular}{|c|c|c|c|}
\hline \multicolumn{4}{|c|}{ Energy $=-916.907234$} \\
\hline $\mathrm{C}$ & 0.82640900 & 1.46715800 & 0.02409300 \\
\hline $\mathrm{C}$ & 1.47986900 & 2.67894000 & 0.05922500 \\
\hline $\mathrm{C}$ & 0.70828300 & 3.87923700 & -0.04187500 \\
\hline $\mathrm{C}$ & -0.65000100 & 3.80543800 & -0.22141800 \\
\hline $\mathrm{C}$ & -1.31119400 & 2.57986100 & -0.24776200 \\
\hline $\mathrm{C}$ & -0.96301200 & 0.10743300 & 0.04793300 \\
\hline $\mathrm{C}$ & 1.21529500 & 0.07863000 & 0.06259200 \\
\hline $\mathrm{H}$ & 2.54688700 & 2.72887700 & 0.17834700 \\
\hline $\mathrm{H}$ & 1.20787900 & 4.83691900 & -0.00907000 \\
\hline $\mathrm{H}$ & -1.24071300 & 4.70048400 & -0.35936600 \\
\hline $\mathrm{H}$ & -2.34901300 & 2.45960700 & -0.49462900 \\
\hline $\mathrm{C}$ & -2.31519600 & -0.39656800 & 0.08451100 \\
\hline $\mathrm{C}$ & -3.40827700 & 0.35832400 & 0.53735700 \\
\hline $\mathrm{C}$ & -2.54437100 & -1.73856200 & -0.30799500 \\
\hline $\mathrm{C}$ & -4.68423200 & -0.15578500 & 0.53627100 \\
\hline $\mathrm{H}$ & -3.24107300 & 1.35026400 & 0.92905600 \\
\hline $\mathrm{C}$ & -3.84343400 & -2.23613700 & -0.33202400 \\
\hline $\mathrm{C}$ & -4.90079200 & -1.45423500 & 0.08066300 \\
\hline $\mathrm{H}$ & -5.50873600 & 0.44230600 & 0.89845500 \\
\hline $\mathrm{H}$ & -3.99261000 & -3.25627600 & -0.65912000 \\
\hline $\mathrm{H}$ & -5.90274000 & -1.86308700 & 0.06730500 \\
\hline $\mathrm{C}$ & 2.51368800 & -0.50967100 & 0.07160100 \\
\hline $\mathrm{C}$ & 3.69083700 & 0.22567200 & -0.17667800 \\
\hline $\mathrm{C}$ & 2.64222900 & -1.89506000 & 0.32219400 \\
\hline $\mathrm{C}$ & 4.92475600 & -0.39408800 & -0.16376500 \\
\hline $\mathrm{H}$ & 3.63858500 & 1.27412700 & -0.41995200 \\
\hline $\mathrm{C}$ & 3.87918700 & -2.49792900 & 0.34091100 \\
\hline $\mathrm{H}$ & 1.75259500 & -2.47716300 & 0.51514300 \\
\hline $\mathrm{C}$ & 5.03223300 & -1.75326000 & 0.10102800 \\
\hline $\mathrm{H}$ & 5.81385300 & 0.18864900 & -0.36767100 \\
\hline $\mathrm{H}$ & 3.95410400 & -3.55833000 & 0.54518100 \\
\hline $\mathrm{H}$ & 6.00303100 & -2.23095400 & 0.11654500 \\
\hline $\mathrm{N}$ & 0.08699500 & -0.69464700 & 0.08420800 \\
\hline $\mathrm{O}$ & -1.55483200 & -2.56717000 & -0.67470000 \\
\hline $\mathrm{H}$ & -0.69073500 & -2.12351000 & -0.47552000 \\
\hline $\mathrm{N}$ & -0.55932600 & 1.43308600 & -0.04538900 \\
\hline
\end{tabular}

